\title{
Revisión y estudio comparativo de la acción del viento sobre las estructuras empleando Eurocó- digo y Código Técnico de la Edificación
}

\author{
A review and comparative study of wind action on \\ structures using Eurocode and the Spanish code (CTE)
}

$\underline{\text { A. Tomás }}^{(*)}$, M. Morales ${ }^{(*)}$

RESUMEN

La publicación del Código Técnico de la Edificación (CTE) en 2006 ha supuesto un salto conceptual en la obtención de la acción del viento sobre las estructuras respecto a la norma básica de acciones anterior. Con el CTE se pretendía adaptar el marco normativo español al programa Eurocódigos, al que debía converger hasta el año 2010. En este contexto se ha realizado un estudio comparativo entre los factores involucrados en la obtención de la acción de viento empleando CTE y Eurocódigo 1. Para cuantificar la influencia de esta acción sobre el diseño estructural se han empleado tres ejemplos de tipologías estructurales habituales: una nave industrial y dos edificios de 5 y 25 plantas. En las conclusiones se aportan comentarios que pueden mejorar la aplicación de la normativa española y se señalan ciertos aspectos contemplados en Eurocódigo 1 que sería recomendable considerar en caso de requerir un diseño estructural más ajustado.

409-14

Palabras clave: Acción del viento; Eurocódigo; Código Técnico de la Edificación; edificación urbana; nave industrial.

\section{SUMMARY}

The publication of the Spanish standard Building Technical Code (CTE) in 2006 was a landmark in obtaining the wind action on structures, with respect to the previous standard. The CTE was developed, among other reasons, to adapt the Spanish standards to the Eurocodes, with which there must be convergence prior to 2010. In this context, a comparative study of the parameters that are involved in obtaining the wind action using the CTE and Eurocode 1 has been carried out. Three examples of conventional structures have been used to quantify the influence of this action on the final design: an industrial building, and two buildings with heights of 5 and 25 storeys. As conclusions of this research, some clarifications to improve the application of Spanish code are provided and some additional capabilities from Eurocode 1 are underlined, which could be considered in order to perform a more precise structural design.
Keywords: Wind action; Eurocode; Spanish code (CTE); urban building; industrial building.

(*) Universidad Politécnica de Cartagena (España). Persona de contacto/Corresponding author: antonio.tomas@upct.es (A. Tomás).

Recibido/Received: 15 jun 2010 Aceptado/Accepted: 12 apr 2012 Publicado online/ Published online: 21 jun 2012 


\section{INTRODUCCIÓN}

La acción del viento sobre las estructuras tiene un papel fundamental en el diseño de las mismas. La complejidad en la descripción del comportamiento turbulento del viento, así como la dificultad para obtener métodos de cuantificación de la carga sobre la estructura a partir de la velocidad característica del viento, han sido tradicionalmente los mayores obstáculos en las sucesivas actualizaciones de la normativa de acciones.

En 1975 se inician los trámites para desarrollar el programa Eurocódigos, mediante el cual se elaboraría un extenso paquete normativo para el proyecto de estructuras. Los principales objetivos del proyecto Eurocódigos son dos: (i) proporcionar técnicas y métodos modernos para el diseño y (ii) homogeneizar el marco normativo europeo. La acción del viento queda incluida en el "Eurocódigo 1: Acciones en estructuras. Parte 1-4: Acciones generales. Acciones de viento" o EC-1 (1).

A nivel internacional, el Eurocódigo referente a cargas de viento se ha posicionado como una de las normas más avanzadas frente a otras como la americana ASCE 7, la canadiense NBCC o la japonesa AIJ. Estudios paramétricos y relativos a la cuantificación de la carga de viento en estas normas se pueden encontrar en la literatura, véase (2) (3) (4) (5) entre otros.

Como fase final del proyecto Eurocódigos, todas las normas nacionales técnicamente divergentes debían anularse antes de finales de marzo de 2010. En esta línea, a nivel nacional se publicó en 2006 el Código Técnico de la Edificación o CTE (6), actualizado por última vez en 2009 según la Orden VIV/984/2009, BOE 23 de abril de 2009, que supuso una destacada evolución respecto a la normativa anterior, la Norma Básica de la Edificación - Acciones en la Edificación de 1988.

En este artículo se expone un estudio comparativo orientado, desde un punto de vista práctico, hacia el técnico que aplica la normativa vigente, y mediante el cual se analizan las posibles diferencias detectadas en el CTE con respecto a EC-1, en lo que se refiere a la obtención de la acción del viento. Estas diferencias se deben, principalmente, al hecho de transponer una norma (EC-1) de aplicación general en edificación y obra civil a otra (CTE) básicamente de aplicación en edificación, así como a diversas erratas de transcripción al pasar de la primera a la segunda. En el estudio se ha realizado una descomposición de la formulación de la carga de viento según ambas normas, identificando los distintos términos y realizando una comparación de los mismos. Finalmente, y de cara a cuantificar la repercusión de estas diferencias en cada parámetro de la carga de viento sobre el diseño global de la estructura, se han empleado tres ejemplos de aplicación de edificación representativa.

\section{FORMULACIÓN DE LA FUERZA DEL VIENTO}

La formulación usada en el CTE tiene su origen en los métodos desarrollados en el EC-1.

\subsection{UNE-EN 1991-1-4 (EC-1)}

La fuerza global actuante sobre una estructura es definida según la siguiente expresión [1].

[1]

$$
F_{V}=C_{s} C_{d} q_{p}\left(z_{e}\right) c_{p} A_{r e f}
$$

El factor estructural $C_{s} C_{d}$ cuantifica el efecto, en las acciones de viento, de la ocurrencia no simultánea de picos de presión sobre la superficie $\left(C_{s^{\prime}}\right.$ factor de escala) así como de las vibraciones estructurales motivadas por la turbulencia del flujo ( $C d$, factor dinámico).

La presión dinámica de pico $q_{p}(z)$ se puede expresar como el producto entre la presión dinámica básica y el coeficiente de exposición [2].

$$
\text { [2] } \quad q_{p}(z)=c_{e}(z) q_{b}=c_{e}(z)\left(\frac{1}{2} \rho v_{b}^{2}\right)
$$

El coeficiente de exposición relaciona la presión dinámica básica (sólo dependiente del clima de la zona) con la presión dinámica de pico o de cálculo, dependiente de la rugosidad del terreno $\left(C_{r}\right)$, de la topografía de la zona $\left(c_{0}\right)$ y de la turbulencia del viento $\left(I_{v}\right)$. El valor de este coeficiente de exposición es [3].

[3]

$$
\begin{aligned}
c_{e}(z)=\frac{q_{p}(z)}{q_{b}} & =\frac{\left[1+7 I_{v}(z)\right]\left[\frac{1}{2} \rho v_{m}^{2}(z)\right]}{\left(\frac{1}{2} \rho v_{b}^{2}\right)}= \\
& =\left\{k_{r}^{2}\left[\ln ^{2}\left(\frac{z}{z_{0}}\right)+7 \ln \left(\frac{z}{z_{0}}\right)\right]\right\}
\end{aligned}
$$

donde $\rho$ es la densidad del aire. La intensidad de la turbulencia $\left(I_{v}\right)$ y la velocidad media del viento a una altura $z\left(v_{m}(z)\right)$, se obtienen según las siguientes expresiones [4].

$$
\begin{aligned}
& I_{v}(z)=\frac{k_{z}}{\ln \left(\frac{z}{z_{0}}\right) c_{o}(z)} \\
& \begin{array}{c}
v_{m}(z)=c_{r}(z) c_{o}(z) v_{b} \\
\operatorname{con} \\
v_{b}=c_{\text {dir }} c_{\text {season }} v_{b, 0}
\end{array}
\end{aligned}
$$


$k_{z}$ es el factor de turbulencia, $z_{0}$ la longitud de la rugosidad, $v_{b}$ la velocidad básica de referencia del viento (independiente de la altura), $k_{r}$ el factor del terreno y $v_{b, 0}$ el valor fundamental de la velocidad básica del viento. El factor direccional $\left(c_{\text {dir }}\right)$ y el estacional $\left(C_{\text {season }}\right)$ tienen en cuenta la influencia de la orientación respecto al viento de la edificación y la estacionalidad de la estructura.

De este modo, la expresión de la fuerza de viento resulta [6].

[6]

$F_{V}=C_{s} C_{d}\left\{k_{r}^{2}\left[\ln ^{2}\left(\frac{z}{z_{0}}\right)+7 \ln \left(\frac{z}{z_{0}}\right)\right]\right\}\left(\frac{1}{2} \rho v_{b}^{2}\right) c_{p} A_{r e f}$

\subsection{CTE DB SE-AE}

En el CTE la carga de viento $F$ sobre una estructura se cuantifica a partir de la siguiente expresión [7].

$$
F_{V}=c_{e} q_{b} c_{p} A
$$

donde $c_{e}$ es el coeficiente de exposición, $q_{b}$ la presión dinámica del viento, $C_{p}$ el coeficiente eólico o de presión y $A$ el área de la estructura expuesta al viento.

El coeficiente de exposición $C_{e}$ y el factor $F$ (en EC-1 $c_{r}(z)$, factor de rugosidad) son [8] [9].

$$
c_{e}=F(F+7 k)
$$

$$
F=k \ln \left(\frac{z}{L}\right)
$$

donde $k$ y $L$ son parámetros característicos de cada tipo de entorno (Tabla D.2 del CTE DB SE-AE).

Agrupando todas las expresiones se llega a [10]:

$F_{V}=\left\{k^{2}\left[\ln ^{2}\left(\frac{z}{L}\right)+7 \ln \left(\frac{z}{L}\right)\right]\right\}\left(\frac{1}{2} \rho v_{b}^{2}\right) c_{p} A$

\section{COMPARACIÓN}

A modo de resumen, se compara en la Tabla 1 la expresión de cada parámetro según las dos normas estudiadas.

El factor de los efectos dinámicos y de escala no se emplea en el CTE, lo que supone adoptar implícitamente un valor unidad para el mismo, fijando un nivel estándar de influencia de estos efectos. Esto puede penalizar el valor de la fuerza de viento en determinados casos, como puede comprobarse en los ejemplos empleados en el apartado 4 , donde $C_{s} C_{d}$ es inferior a la unidad.
Tabla 1. Resumen de parámetros de cálculo de la fuerza del viento según EC-1 y CTE

\begin{tabular}{|c|c|c|c|c|c|}
\cline { 2 - 6 } \multicolumn{1}{c|}{} & $\begin{array}{c}\text { Presión } \\
\text { dinámica básica } \\
\text { del viento }\end{array}$ & $\begin{array}{c}\text { Coeficiente } \\
\text { de } \\
\text { exposición }\end{array}$ & $\begin{array}{c}\text { Coeficiente } \\
\text { de } \\
\text { arrastre }\end{array}$ & $\begin{array}{c}\text { Área } \\
\text { expuesta } \\
\text { al viento }\end{array}$ & $\begin{array}{c}\text { Efectos } \\
\text { dinámicos } \\
\text { y de escala }\end{array}$ \\
\hline EC-1 & $\left(\frac{1}{2} \rho v_{b, 0}^{2}\right)$ & $k_{r}^{2}\left[\ln ^{2}\left(\frac{z}{z_{0}}\right)+7 \ln \left(\frac{z}{z_{0}}\right)\right]$ & $C_{p}$ Ó $C_{f}$ & $A_{\text {ref }}$ & $C_{s} C_{d}$ \\
\hline CTE & $\left(\frac{1}{2} \rho v_{b}^{2}\right)$ & $k^{2}\left[\ln ^{2}\left(\frac{z}{L}\right)+7 \ln \left(\frac{z}{L}\right)\right]$ & $C_{p}$ & $A$ & - \\
\hline
\end{tabular}

A continuación se analiza cada parámetro de la carga de viento según el CTE y el EC-1, con el fin de estudiar las posibles divergencias existentes.

\subsection{Categorías de rugosidad del terreno}

La categoría de rugosidad del terreno permite obtener, en función del entorno, la carga de viento sobre una estructura o elemento estructural. La rugosidad del terreno, caracterizada por el valor de la longitud de rugosidad zo, modifica el perfil de velocidad a bajas alturas en función del nivel medio de obstáculos.

EC-1 define cinco categorías diferentes, desde la 0 a la IV (Tabla 2).

Tabla 2. Categorías y parámetros del terreno según EC-1

\begin{tabular}{|c|c|c|c|}
\hline $\begin{array}{c}\text { Nombre de } \\
\text { la categoría }\end{array}$ & Descripción del terreno & $\mathbf{z}_{0}$ & $\mathbf{z}_{\min }$ \\
\hline Categoría 0 & $\begin{array}{c}\text { Mar abierto o zona costera } \\
\text { expuesta al mar abierto. }\end{array}$ & 0,003 & 1 \\
\hline Categoría I & $\begin{array}{c}\text { Lagos o áreas planas y horizontales con } \\
\text { vegetación despreciable y sin obstáculos. }\end{array}$ & 0,01 & 1 \\
\hline Categoría II & $\begin{array}{c}\text { Áreas con vegetación baja (hierba) y } \\
\text { obstáculos aislados, con separación mayor de } \\
\text { 20 veces la altura de los obstáculos. }\end{array}$ & 0,05 & 2 \\
\hline Categoría III & $\begin{array}{c}\text { Áreas con cobertura de vegetación uniforme o } \\
\text { edificación con obstáculos aislados de separación } \\
\text { máxima de 20 veces la altura de los obstáculos. }\end{array}$ & 0,3 & 5 \\
\hline Categoría IV & $\begin{array}{c}\text { Áreas en las que al menos un 15 \% } \\
\text { de la superficie está cubierta por edificios cuya } \\
\text { altura media supera los 15 m. }\end{array}$ & 1 & 10 \\
\hline
\end{tabular}

En el CTE, a las categorías se les denomina grados de aspereza. Son también cinco, numeradas en este caso desde la I a la V (Tabla 3).

Tabla 3. Categorías y parámetros del terreno según CTE

\begin{tabular}{|c|c|c|c|c|}
\hline $\begin{array}{c}\text { Nombre de } \\
\text { la categoría } \\
\text { (grado de aspereza) }\end{array}$ & Descripción del terreno & $z_{0}$ & $z_{\min }$ & $k$ \\
\hline Categoría I & $\begin{array}{c}\text { Borde del mar o de un lago, con una superficie } \\
\text { de agua en la dirección del viento } \\
\text { de al menos 5 km de longitud }\end{array}$ & 0,003 & 1 & 0,156 \\
\hline Categoría II & $\begin{array}{c}\text { Terreno rural Ilano sin obstáculos } \\
\text { ni arbolado de importancia }\end{array}$ & 0,01 & 1 & 0,17 \\
\hline Categoría III & $\begin{array}{c}\text { Zona rural accidentada o Ilana } \\
\text { con algunos obstáculos aislados, }\end{array}$ & 0,05 & 2 & 0,19 \\
\hline Categoría IV & $\begin{array}{c}\text { Zono árboles o construcciones pequeñas } \\
\text { industrial o forestal }\end{array}$ & 0,3 & 5 & 0,22 \\
\hline Categoría V & $\begin{array}{c}\text { Centro de negocios de grandes ciudades, } \\
\text { con profusión de edificios en altura }\end{array}$ & 1 & 10 & 0,24 \\
\hline
\end{tabular}


1. Factor de rugosidad en función de la altura para las cinco categorías de terreno según EC-1.
Estas categorías, o grados de aspereza del CTE, están adoptadas de EC-1, pero se ha modificado la descripción de las mismas intentando adaptarlas al ámbito edificatorio. Aunque estas descripciones ocasionen, en general, interpretaciones adecuadas, es cierto que podría surgir alguna incongruencia al realizar una interpretación subjetiva. Por ejemplo, una "Zona urbana en general" (categoría IV en CTE), fácilmente puede ser un "Área en la que al menos un $15 \%$ de la superficie esté cubierta por edificios cuya altura media supera los 15 m" (categoría IV en EC-1), por lo que se originaría una caracterización matemática distinta del mismo entorno, y en consecuencia distintos valores finales de la carga de viento. un perfil logarítmico de velocidad (Figura 1), adoptando el valor unidad para la citada altura de 10 m y categoría de terreno II, es decir, la velocidad básica sobre la que se aplica este coeficiente debe estar referida a dicha categoría de terreno II (EC-1) o III (CTE).

\subsection{Velocidad de referencia del viento}

La velocidad de referencia del viento es función del clima característico de una región y se define para una determinada categoría de terreno. Se ha empleado la categoría II de EC-1 como referencia para homogeneizar los valores de velocidad propuestos por cada norma y poder compararlos posteriormente. Las categorías empleadas en la distinta normativa se muestran en la Tabla 4.

Tabla 4. Categorías de terreno del viento de referencia según distinta normativa

\begin{tabular}{|c|c|c|c|c|}
\cline { 2 - 5 } \multicolumn{1}{c|}{} & Categoría & Descripción & $Z_{0}$ & $Z_{\min }$ \\
\hline EC-1 & II & $\begin{array}{c}\text { Áreas con vegetación baja (hierba) y } \\
\text { obstáculos aislados, con separación mayor } \\
\text { de 20 veces la altura de los obstáculos }\end{array}$ & 0,05 & 2 \\
\hline ROM 0.4-95 & I & $\begin{array}{c}\text { Mar abierto y campo abierto } \\
\text { Ilano sin obstáculos }\end{array}$ & 0,005 & 0 \\
\hline IAPF-07 / IAP-11 & II & $\begin{array}{c}\text { Zona rural accidentada o llana } \\
\text { con algunos obstáculos aislados, } \\
\text { como árboles o construcciones pequeñas }\end{array}$ & 0,05 & 2 \\
\hline
\end{tabular}

El coeficiente que tiene en cuenta este efecto es el factor de rugosidad $\left(c_{r}\right.$ en EC- $1, F$ en CTE). Ambos dependen de $z_{0}, z_{\min } y$ del factor del terreno ( $k_{r}$ en EC-1, $k$ en CTE). Este factor sólo se puede calcular explícitamente en EC-1, apareciendo en CTE tabulado (Tabla 3).

La relación entre el valor básico de la velocidad del viento $v_{b^{\prime}}$ medido en la categoría II (grado de aspereza de referencia) a una altura de $10 \mathrm{~m}$, y la velocidad del viento a una altura $z$ en un terreno de diferente aspereza, se puede expresar mediante el citado factor de rugosidad $C_{r}$ (factor $F$ en CTE). Representa

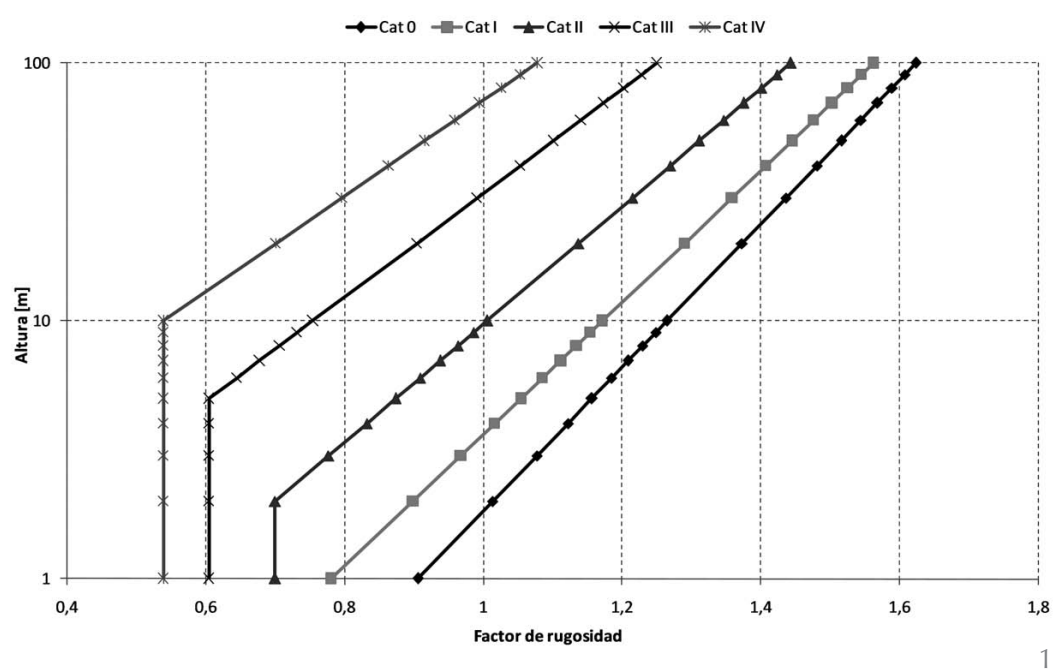

Se ha comparado la velocidad de referencia proporcionada en las normas ROM (7), CTE (6), IAP-11 (8) e IAPF-07 (9). El mapa de la ROM está muy detallado, mientras que tanto CTE como IAP-11 e IAPF-07 presentan mapas mucho más sencillos, con menor número de zonas.

\subsubsection{ROM 0.4-95}

En el Anejo I de ROM se describen las características de la estimación de la velocidad de referencia del viento. Los valores de velocidad de ROM que aparecen en el mapa deben corregirse para ser expresados en categoría de rugosidad II. Más detalles pueden consultarse en (10) (11).

\subsubsection{CTE}

En el Código Técnico de la Edificación se recoge un mapa de velocidad de referencia del viento, definido en categoría III (categoría II de EC-1). En este caso la zonificación se hace en tres regiones de diferente velocidad de referencia del viento: 26,27 y $29 \mathrm{~m} / \mathrm{s}$.

\subsubsection{IAPF-07 / IAP-11}

La instrucción IAPF-07 clasifica el territorio español en dos regiones de distinta velocidad, 24 y $28 \mathrm{~m} / \mathrm{s}$, mientras que la IAP11 adopta el mapa de isotacas del CTE. 
La IAPF-07 se basa en la ROM para zonas costeras y en datos de estaciones meteorológicas para el interior (12). Se detecta una incongruencia en cuanto al entorno de referencia en el que se encuadran los valores de velocidad, pues en ambas instrucciones se describe un terreno de categoría "zona plana y desprotegida frente al viento" que a continuación se afirma que es equivalente a categoría II (zona rural con vegetación baja y obstáculos aislados), no quedando entonces claro qué tipo de terreno es el de referencia. Se ha asumido, para los cálculos, la categoría II como categoría de referencia, pues se obtienen valores en concordancia con los del resto de normas.

\subsubsection{AEMET}

Para cuantificar la precisión de la velocidad de viento proporcionada por CTE para una zona de España, en este caso para la Región de Murcia que es donde se ha realizado este estudio, se ha adquirido información sobre la velocidad del viento a la Agencia Estatal de Meteorología (AEMET).

En la Región de Murcia existen un total de 16 estaciones. Desechando las que no proporcionan la velocidad máxima media de viento en $10 \mathrm{~min}$, y las que tienen un tamaño muestral de menos de 10 años, resultan las estaciones de la Tabla 5.

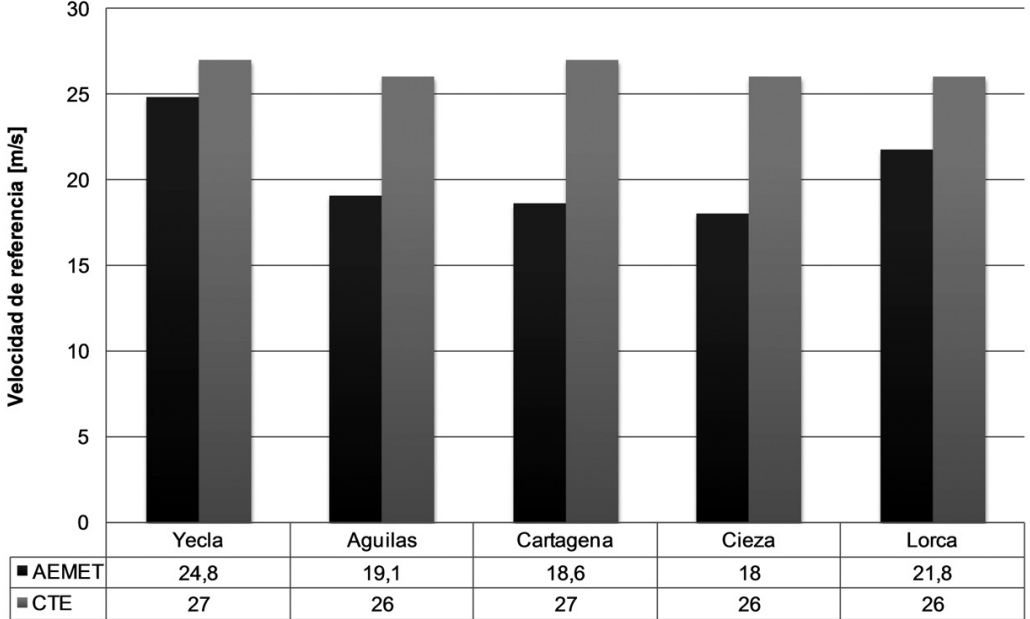

\section{2}

Además, en todos los casos los valores de velocidad de CTE son conservadores respecto a los medidos en las estaciones.

\subsubsection{Comparación}

En la Figura 3 se muestran los valores de velocidad propuestos por cada norma para diferentes ciudades españolas. Se aprecia un buen acuerdo entre IAPF-07 y CTE / IAP-11, con mapas de viento obtenidos con los datos más recientes de la AEMET (anteriormente INM). Los valores propuestos por ROM 0.4-95 son sensiblemente menores, teniendo en cuenta que su zonificación es mucho más detalla.
2. Comparativa entre la velocidad básica de referencia del viento propuesta por CTE y la elaborada mediante datos de estaciones de medida de la AEMET, para cinco poblaciones de la Región de Murcia.

3. Velocidad básica de referencia del viento según IAPF-07, CTE IAP-11 y ROM 0.4-95, para diferentes ciudades españolas.

Tabla 5. Datos de las estaciones de medida de viento relevantes en la Región de Murcia

\begin{tabular}{|c|c|c|c|c|c|}
\hline $\begin{array}{c}\text { Referencia } \\
\text { (AEMET) }\end{array}$ & $\begin{array}{c}\text { Nombre de } \\
\text { la estación }\end{array}$ & $\begin{array}{c}\text { Altitud } \\
{[\mathbf{m}]}\end{array}$ & $\begin{array}{c}\text { Fecha } \\
\text { de alta }\end{array}$ & Longitud & Latitud \\
\hline 7012 C & CARTAGENA (CIUDAD) & 17 & $01 / 09 / 1988$ & 59122 & 373608 \\
\hline $7145 D$ & CIEZA (P. BOMBEROS) & 265 & $01 / 02 / 1997$ & 122582 & 381408 \\
\hline 7209 & LORCA (C.C.A.) & 320 & $01 / 01 / 1953$ & 141222 & 373912 \\
\hline $7002 X$ & AGUILAS (P. BOMBEROS) & 6 & $01 / 05 / 1997$ & 135095 & 372453 \\
\hline $7275 B$ & YECLA (COOP. FRUTAS) & 590 & $01 / 11 / 1969$ & 106152 & 383630 \\
\hline
\end{tabular}

Fuente: AEMET.

Las dos distribuciones estadísticas que mejor se ajustan al comportamiento de las tormentas de viento, y en consecuencia a la velocidad del viento, son la distribución extrema de tipo I o de Weibull y la de Gumbel (13) (14). Ambas distribuciones predicen velocidades de referencia muy similares, por lo que se ha optado por realizar la predicción con la de tipo Weibull. Se puede encontrar el procedimiento con más detalle en (11).

En la Figura 2 se expone el valor característico del viento con una probabilidad de excedencia del $2 \%$ (período de retorno de 50 años) para cada estación meteorológica analizada. También se muestran las velocidades correspondientes empleando el mapa de viento de CTE. Las diferencias existentes entre los valores de AEMET y CTE son aceptables, teniendo en cuenta que el mapa de CTE discretiza todo el territorio nacional en tres rangos de velocidad.

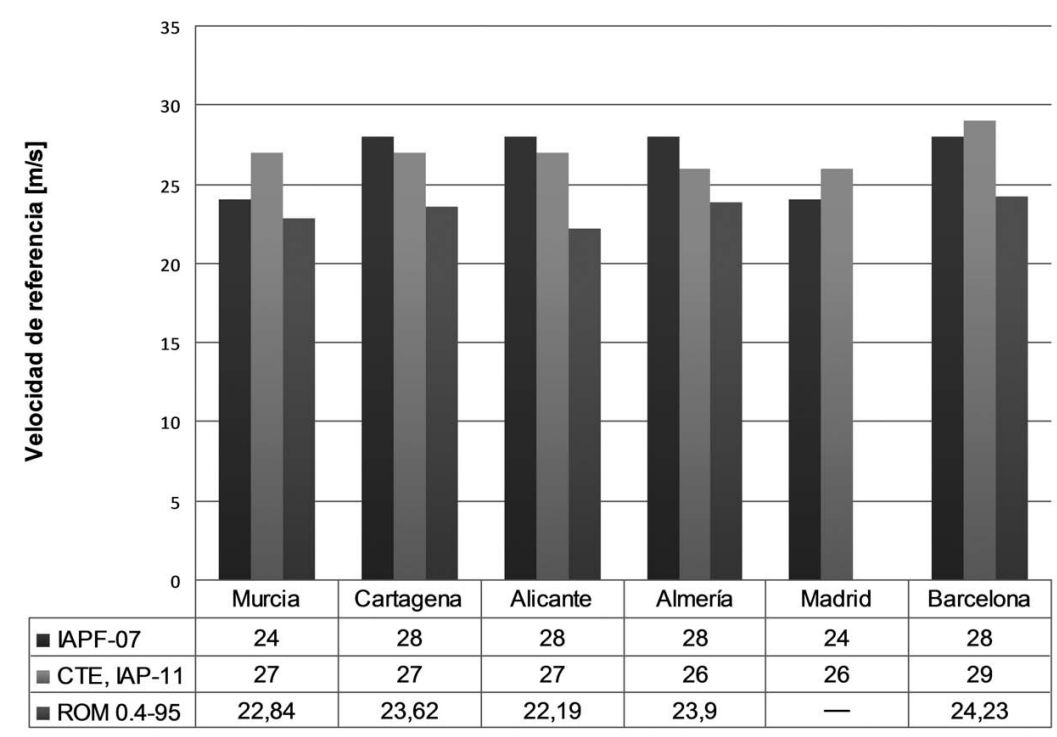




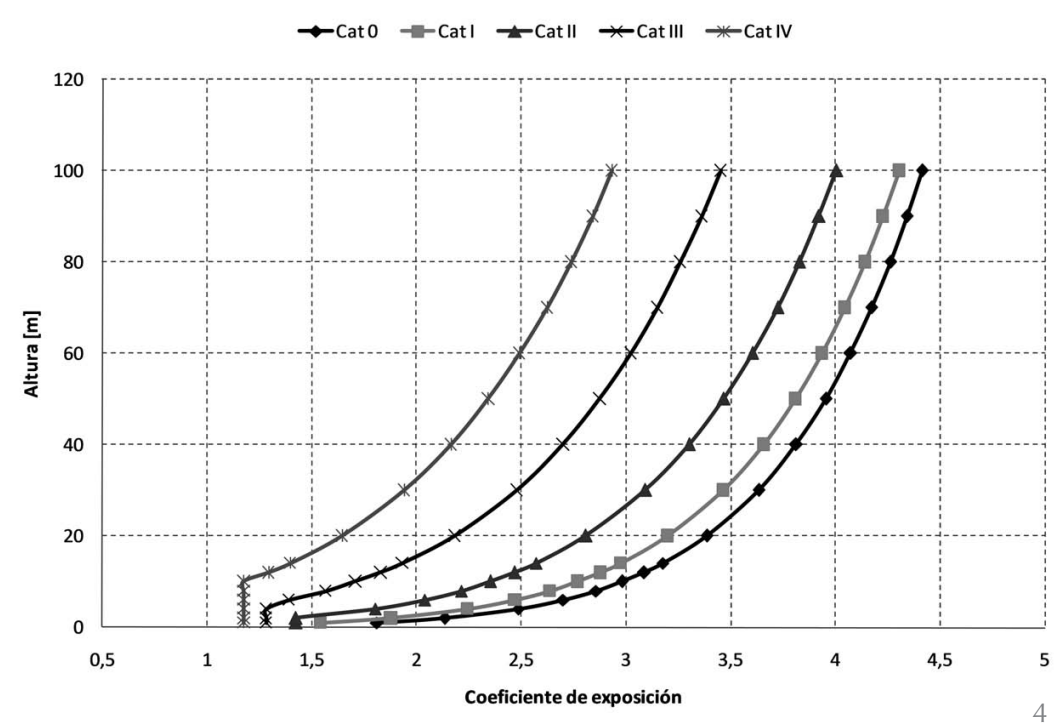

4. Coeficiente de exposición en función de la altura para las cinco categorías de terreno de EC-1.

\subsection{Coeficiente de exposición}

\subsubsection{EN 1991-1-4 (EC-1)}

Para representar gráficamente el coeficiente de exposición de EC-1, se han usado los valores del factor del terreno redondeados al cuarto decimal. Para valores desde $z=0 \mathrm{~m}$ hasta $z=100 \mathrm{~m}$, la gráfica del coeficiente de exposición para las cinco categorías de terreno definidas en EC- 1 es la mostrada en la Figura 4.

Una vez combinado el coeficiente de exposición con la presión dinámica básica - para obtener la presión dinámica de cálculo -, y dependiendo de las dimensiones relativas del edificio analizado, el apartado 7.2.2 de EC-1 propone distintas distribuciones del perfil de presiones. El valor de la altura de referencia $z_{e}$ es distinto en cada caso y dependiente del tramo de elevación al que se

Tabla 6. Coeficiente del terreno $k$ según CTE y según EC-1 (redondeado a dos y cuatro decimales)

\begin{tabular}{|c|c|c|c|c|}
\hline \multirow{2}{*}{ CTE } & \multirow{2}{*}{ EC-1 } & \multicolumn{3}{|c|}{ Factor del terreno (k) } \\
\cline { 3 - 5 } & & CTE & $\begin{array}{c}\text { EC-1 } \\
\text { (redondeo 4 decimales) }\end{array}$ & $\begin{array}{c}\text { EC-1 } \\
\text { (redondeo 2 decimales) }\end{array}$ \\
\hline I & 0 & 0,156 & 0,1560 & 0,16 \\
\hline II & I & 0,17 & 0,1697 & 0,17 \\
\hline III & II & 0,19 & 0,1900 & 0,19 \\
\hline IV & III & 0,22 & 0,2154 & 0,22 \\
\hline V & IV & 0,24 & 0,2343 & 0,23 \\
\hline
\end{tabular}

Tabla 7. Incremento porcentual del coeficiente de exposición de CTE respecto a EC-1, para cada categoría de exposición y para diferentes alturas de construcción

\begin{tabular}{|l|c|c|c|c|c|}
\hline \multicolumn{1}{|c|}{$\begin{array}{c}\text { Descripción de la } \\
\text { categoría del terreno }\end{array}$} & CTE & EC-1 & $\begin{array}{c}\text { Nave industrial } \\
\boldsymbol{h = 6 \mathbf { m }}\end{array}$ & $\begin{array}{c}\text { Edificio } \\
\mathbf{5} \text { plantas } \\
\boldsymbol{h}=\mathbf{1 7 , 5} \mathbf{m}\end{array}$ & $\begin{array}{c}\text { Edificio } \\
\mathbf{2 5} \text { plantas } \\
\boldsymbol{h}=\mathbf{1 0 0} \mathbf{~ m}\end{array}$ \\
\hline Borde de mar (1) & I & 0 & 0,00 & 0,00 & 0,00 \\
\hline $\begin{array}{l}\text { Terreno rural Ilano } \\
\text { sin obstáculos aislados (2) }\end{array}$ & II & I & 0,35 & 0,35 & 0,35 \\
\hline $\begin{array}{l}\text { Zona rural con } \\
\text { obstáculos aislados (3) }\end{array}$ & III & II & 0,00 & 0,00 & 0,00 \\
\hline $\begin{array}{l}\text { Superficies boscosas, } \\
\text { pequeñas zonas urbanas (4) }\end{array}$ & IV & III & 4,32 & 4,32 & 4,32 \\
\hline $\begin{array}{l}\text { Centro de grandes } \\
\text { ciudades (5) }\end{array}$ & V & IV & 4,92 & 4,92 & 4,92 \\
\hline
\end{tabular}

aplique, disminuyendo la carga de viento sobre la fachada respecto del perfil uniforme usado en CTE. Esto se puede apreciar gráficamente en uno de los ejemplos de aplicación del apartado 4, concretamente en la Figura 6.

\subsubsection{CTE DB SE-AE}

La formulación para calcular el valor del coeficiente de exposición en CTE es idéntica a la de EC-1, excepto que en CTE la altura de referencia $z_{\text {ref }}$ para calcular la presión dinámica de cálculo, es constante para cualquier valor de $z$ e igual a la altura total del edificio. CTE no aporta, como hace EC-1, una expresión para calcular el factor del terreno $k$, sino que lo presenta de forma tabulada (Tabla 6), con cuatro de sus cinco valores redondeados al segundo decimal (6). Uno de esos valores, el correspondiente a categoría $\mathrm{V}$, aparece redondeado por exceso, cuando debería ser por defecto.

En el CTE, el coeficiente de exposición se puede obtener de dos formas: (i) empleando las expresiones del Anejo D, o (ii) empleando la Tabla 3.4 (cuyos valores proceden de aplicar dichas expresiones para una serie de alturas determinadas hasta $30 \mathrm{~m}$ ).

\subsubsection{Comparación}

Considerando como referencia la fórmula del factor del terreno que aparece en la versión definitiva de EC-1 (redondeado a 4 decimales), se realiza la comparación del coeficiente de exposición de CTE respecto a EC-1. La Tabla 7 recoge la diferencia porcentual entre ellas. Se ha estudiado la variación para tres alturas diferentes, correspondientes a alturas típicas de construcción (nave industrial, edificio de 5 plantas y edificio de 25 plantas).

Las escasas diferencias surgidas entre CTE y EC-1 se deben al número de decimales usados en la obtención del factor del terreno $k$ y al hecho de redondear por exceso la categoría $\vee$ en CTE en vez de por defecto.

\subsection{Presión dinámica de cálculo}

La expresión de la presión dinámica de cálculo coincide con la expresión de la presión dinámica de pico según la ecuación [2]. Tanto la velocidad de referencia como el coeficiente de exposición están presentes en la expresión, por lo que ambos deben estar referenciados a la misma categoría de rugosidad del terreno. Al comparar los valores de la presión dinámica de cálculo, las diferencias se deben al factor de rugosidad del terreno, por lo que coinciden con los valores de la Tabla 7. 


\subsection{Coeficientes de presión exterior}

El CTE presenta los mismos valores de coeficientes de presión que EC-1. Afectan a todas las superficies exteriores de la construcción, estando zonificados sobre dichas superficies y dependiendo su valor del área del elemento solicitado. CTE no contempla la reducción de hasta un 15\% como máximo de las fuerzas sobre paramentos verticales a barlovento y a sotavento debido a la falta de correlación de presiones.

\subsection{Factor estructural $C_{s} C_{d}$}

Este factor, recogido sólo en EC-1, se emplea para considerar los efectos dinámicos del viento. Sus valores están comprendidos entre 0,85 y 1,15 , adoptando de forma simplificada el valor unidad en los casos más habituales.

Si se desea una mayor precisión en la obtención del factor estructural se puede emplear los métodos existentes en EC-1, Anexos B y C. Para estructuras de altas frecuencias será menor que 1 , con un mínimo de 0,85 . En el caso de bajas frecuencias será mayor que 1 . En los cálculos de este estudio se ha empleado el método propuesto en el Anexo C. Puede consultarse con más detalle en la referencia (11).

\section{EJEMPLOS DE APLICACIÓN}

A continuación se procede a analizar tres construcciones tipo, con el fin de evaluar la repercusión de la carga de viento sobre el diseño estructural según CTE y EC-1:

1. Nave industrial (Figura 5a). Compuesta por 11 pórticos, cubierta a dos aguas con una pendiente del $10 \%, 22 \mathrm{~m}$ de luz, $50 \mathrm{~m}$ de longitud y $5 \mathrm{~m}$ de altura de pilares. Representa un caso habitual de nave industrial con estructura metálica.

2. Edificio de 5 plantas (Figura 5b). Dimensiones en planta $24 \times 24 \mathrm{~m}$, altura entreplantas de 3,5 m, estructura aporticada en cada dirección con 5 vanos. Representa un caso habitual de edificio de viviendas con estructura de hormigón.

3. Edificio de 25 plantas (Figura $5 \mathrm{C}$ ). Dimensiones en planta $24 \times 24 \mathrm{~m}$, altura entreplantas de $4 \mathrm{~m}$, estructura aporticada en cada dirección con 3 vanos. Representa el caso extremo de CTE (valor máximo de esbeltez). La estructura se ha supuesto de hormigón. Incorpora el perfil del coeficiente de exposición reducido en función de la altura (Figura 6).

En cada uno de los tres modelos descritos, se procede a comparar los estados tensionales, consecuencia de aplicar las acciones consi-

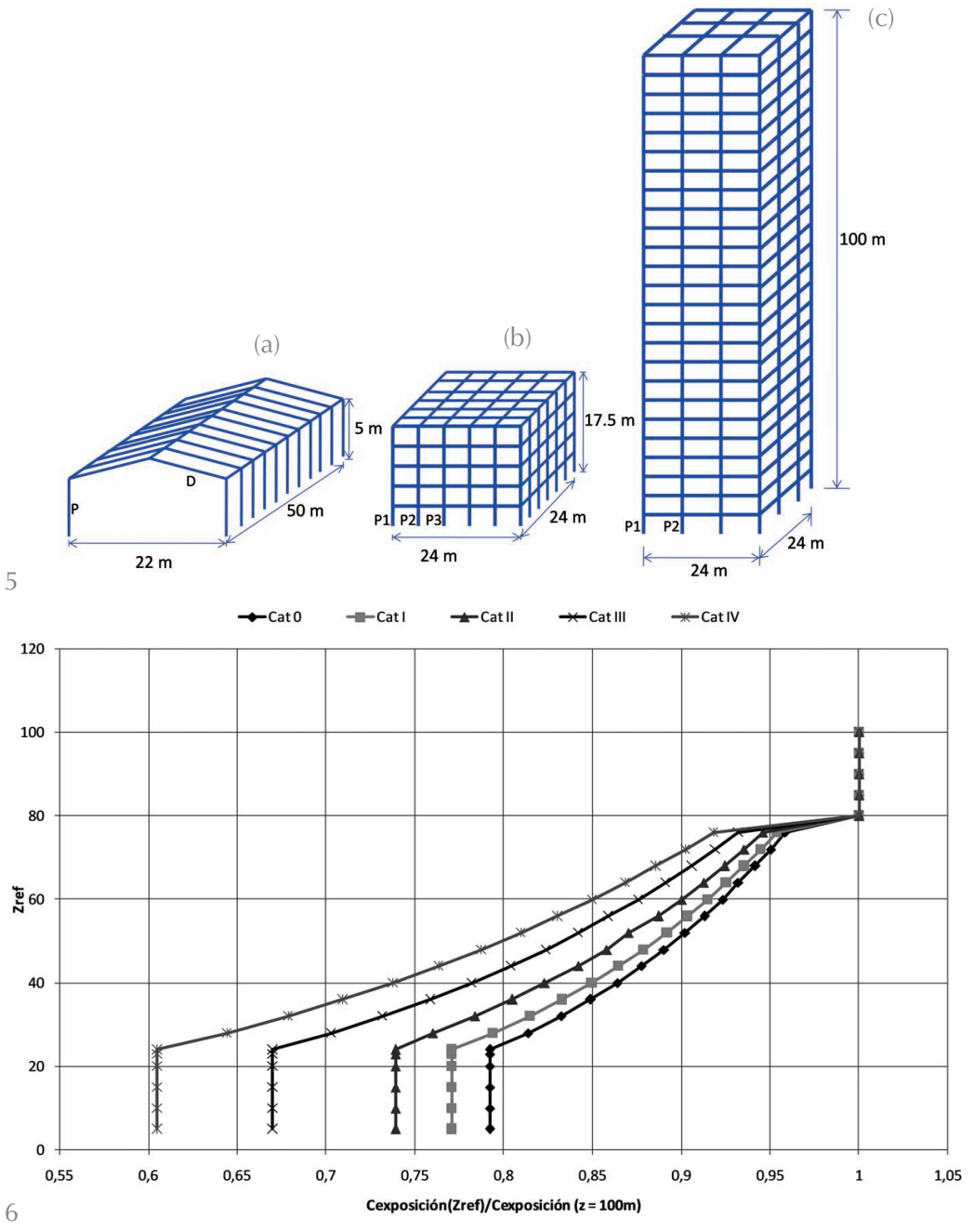

deradas en el CTE (carga muerta, sobrecarga de uso para conservación -no concomitante con el resto de acciones variables-, carga de nieve y carga de viento) teniendo en cuenta que, para el modelo identificado como CTE, la carga de viento se estima según el CTE, mientras que, para el modelo identificado como EC-1, se estima según el EC-1.

Respecto a la carga de viento, en ambos casos, CTE y EC-1, se han supuesto los paramentos zonificados según cada norma. La carga puntual aplicada a cada nudo de la estructura es el producto de la carga por unidad de superficie proporcionado por cada norma multiplicada por el área tributaria del nudo. La carga de viento de las regiones $\mathrm{D}$ y $\mathrm{E}$, tanto en los modelos CTE como EC-1, se considera soportada uniformemente por todos los pórticos que existen en cada modelo estructural en esa dirección. La identificación de los distintos casos calculados según estos dos modelos se realiza mediante la combinación de dos caracteres: una cifra, del 1 al 5, asociada a cada categoría de exposición (Tabla 7), junto con una letra $(\mathrm{a}, \mathrm{b}, \mathrm{c})$ asociada a cada una de las tres regiones geográficas $(A, B, C)$ del mapa de viento de CTE. Así por ejemplo, un modelo 
7. Nave industrial. Incremento de tensión de Von Misses en pilares y dintel. CTE respecto a EC-1. identificado como $1 \mathrm{~b}$ se ve afectado por la exposición "Borde de mar" y se encuentra situado dentro de la zona geográfica B.

Se ha considerado como representativa del estado estructural la tensión equivalente de Von Misses máxima, para cada estado de carga y norma. En el caso de la nave, los elementos de referencia son los pilares y el dintel, mientras que en el caso del edificio son los pilares de la base. En cada modelo estructural se han empleado los mismos perfiles para comparar las tensiones equivalentes, tanto CTE como EC-1, desde 1A hasta 5C. De todos los pórticos que tiene cada modelo de edificio, se adoptan como representativos dos, uno exterior y otro interior, por ejemplo, 1A_e y 1A_i. El análisis se ha realizado en el programa DISSENY $(15,16)$.
- Reducción de la altura de referencia, para calcular el coeficiente de exposición, de hasta un $39 \%$ en el caso del edificio de 25 plantas (Figura 6).

En las Figuras 7 a 9 se muestra el incremento de tensiones, en los elementos principales de cada estructura, bajo el modelo de cargas propuesto en el CTE respecto a EC-1. Los valores de $C_{s} C_{d}$ empleados en el cálculo de cada modelo se recogen en la Tabla 8.

\subsection{Nave Industrial}

En la Figura 7 se muestran los incrementos de tensión entre los modelos de CTE y de EC-1 para el caso de la nave industrial.

Tabla 8. Factor estructural $C_{s} C_{d}$ de cada modelo, según categoría del terreno y velocidad de referencia del viento

\begin{tabular}{|c|c|c|c|c|c|c|c|c|c|c|c|}
\hline \multicolumn{3}{|c|}{$\begin{array}{c}\text { Categoría } \\
\text { de terreno }\end{array}$} & \multicolumn{3}{|c|}{$\begin{array}{c}\text { Nave } \\
\text { Industrial }\end{array}$} & \multicolumn{3}{|c|}{$\begin{array}{l}\text { Edificio de } \\
5 \text { plantas }\end{array}$} & \multicolumn{3}{|c|}{$\begin{array}{l}\text { Edificio de } \\
25 \text { plantas }\end{array}$} \\
\hline & $z$ & Z & Zona A & Zona B & Zona C & Zona A & Zona B & Zona C & Zona A & Zona B & Zona C \\
\hline \multirow{2}{*}{0} & \multirow{2}{*}{0,003} & \multirow{2}{*}{1} & 0,9645 & 0,9665 & 0,9703 & \multirow{2}{*}{0,9464} & \multirow{2}{*}{0,9493} & \multirow{2}{*}{0,9539} & \multirow{2}{*}{0,9505} & \multirow{2}{*}{0,9525} & \multirow{2}{*}{0,9561} \\
\hline & & & 0,9416 & 0,9437 & 0,9475 & & & & & & \\
\hline \multirow{2}{*}{ I } & \multirow{2}{*}{0,01} & \multirow{2}{*}{1} & 0,9552 & 0,9548 & 0,959 & \multirow{2}{*}{0,9370} & \multirow{2}{*}{0,9393} & \multirow{2}{*}{0,9437} & \multirow{2}{*}{0,9396} & \multirow{2}{*}{0,9417} & \multirow{2}{*}{0,9485} \\
\hline & & & 0,9282 & 0,9305 & 0,9349 & & & & & & \\
\hline \multirow{2}{*}{ II } & \multirow{2}{*}{0,05} & \multirow{2}{*}{2} & 0,926 & 0,928 & 0,934 & \multirow{2}{*}{0,9174} & \multirow{2}{*}{0,9199} & \multirow{2}{*}{0,9246} & \multirow{2}{*}{0,9194} & \multirow{2}{*}{0,9217} & \multirow{2}{*}{0,9262} \\
\hline & & & 0,899 & 0,9018 & 0,9078 & & & & & & \\
\hline \multirow{2}{*}{ III } & \multirow{2}{*}{0,3} & \multirow{2}{*}{5} & 0,8902 & 0,8902 & 0,8913 & \multirow{2}{*}{0,8964} & \multirow{2}{*}{0,8964} & \multirow{2}{*}{0,8965} & 09083 & & \\
\hline & & & 0,886 & 0,886 & 0,886 & & & & 0,9003 & & \\
\hline II & 1 & 10 & 0,885 & 0,885 & 0,885 & 08848 & 08848 & 08848 & 08993 & 08995 & 08999 \\
\hline & & & 0.882 & 0.882 & 0.882 & & & & & & \\
\hline
\end{tabular}

Al seguir el procedimiento comentado y al comparar los resultados de CTE con EC-1 se está valorando la influencia de:

- Categorías de exposición: Excesivo redondeo del factor del terreno.

- Introducción del factor estructural $C_{s} C_{d}$.

- Reducción del $15 \%$ de la fuerza total equivalente actuando sobre los paramentos verticales situados a barlovento y a sotavento debido a la falta de correlación de las presiones.
El valor unidad significa que no existe diferencia entre ambos, por tanto la hipótesis de combinación de cargas que condiciona el diseño no depende de la carga de viento.

De la Figura 7 se desprende que no existen diferencias en los dinteles de los pórticos interiores (la carga de viento no está incluida en la hipótesis de cargas dominante), mientras que en los pilares existe una diferencia menor al 5\%. En el caso de los pórticos exteriores, las diferencias, tanto en

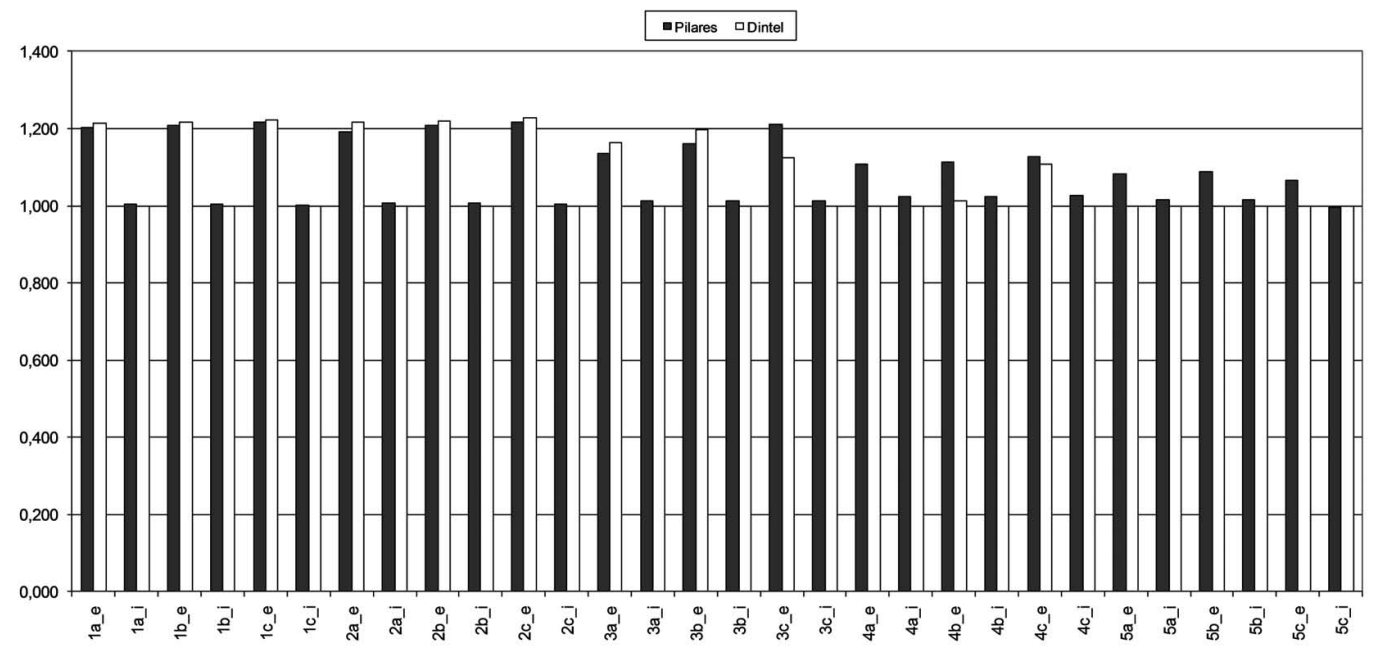


pilares como en dinteles, son de aproximadamente el $20 \%$ para las categorías 1,2 y 3 , e inferiores al $10 \%$ para las categorías 3 a 5. La aplicación del factor estructural $C_{s} C_{d \prime}$ junto con la reducción del $15 \%$ de la fuerza total por la falta de correlación entre las presiones a barlovento y a sotavento, contribuyen por igual a estas diferencias finales.

\subsection{Edificio de 5 plantas.}

Debido a la simetría del modelo, sólo se necesita representar tres de los cinco pilares que conforman cada pórtico del modelo del edificio de 5 plantas. En la gráfica de las Figura 8, P1 se corresponde con el pilar exterior, P2 con el intermedio y P3 con el central. Se observan diferencias similares entre los pilares P1, P2 y $\mathrm{P} 3$, por lo que no se distingue entre ellos al describir los resultados.

Se aprecia una diferencia algo superior al $10 \%$ en pórticos interiores y al $15 \%$ en pórticos exteriores. De forma general, el factor $C_{s} C_{d}$ y la reducción del $15 \%$ de la fuerza total -por la falta de correlación entre las presiones a barlovento y a sotavento- originan la mayor parte de esas diferencias.

\subsection{Edificio de 25 plantas}

Del mismo modo que en el ejemplo de edificio de 5 plantas, es posible representar el comportamiento del modelo de 25 plantas usando sólo dos de los cuatro pilares de cada pórtico. En la gráfica de la Figura 9, P1 se corresponde con el pilar exterior y $\mathrm{P} 2$ con el interior.

Nuevamente, se observan diferencias similares entre los pilares P1 y P2, y los resultados para el pórtico interior y para un pórtico exterior se pueden comentar siguiendo las mismas directrices. En general, se aprecian diferencias de entre un $20 \%$ y un $35 \%$ de incremento.

Fundamentalmente, la presión dinámica de pico reducida en EC-1, junto al efecto de no usar el factor $C_{s} C_{d}$, origina entre un $10 \%$ y un $25 \%$ más de tensiones empleando el CTE, pues ambos factores relajan la carga de viento conforme el entorno sea de menor categoría.
8. Edificio de 5 plantas. Incremento de tensión de Von Misses en pilares. CTE respecto a EC-1.

9. Edificio de 25 plantas. Incremento de tensión de Von Misses en pilares. CTE respecto a EC-1.
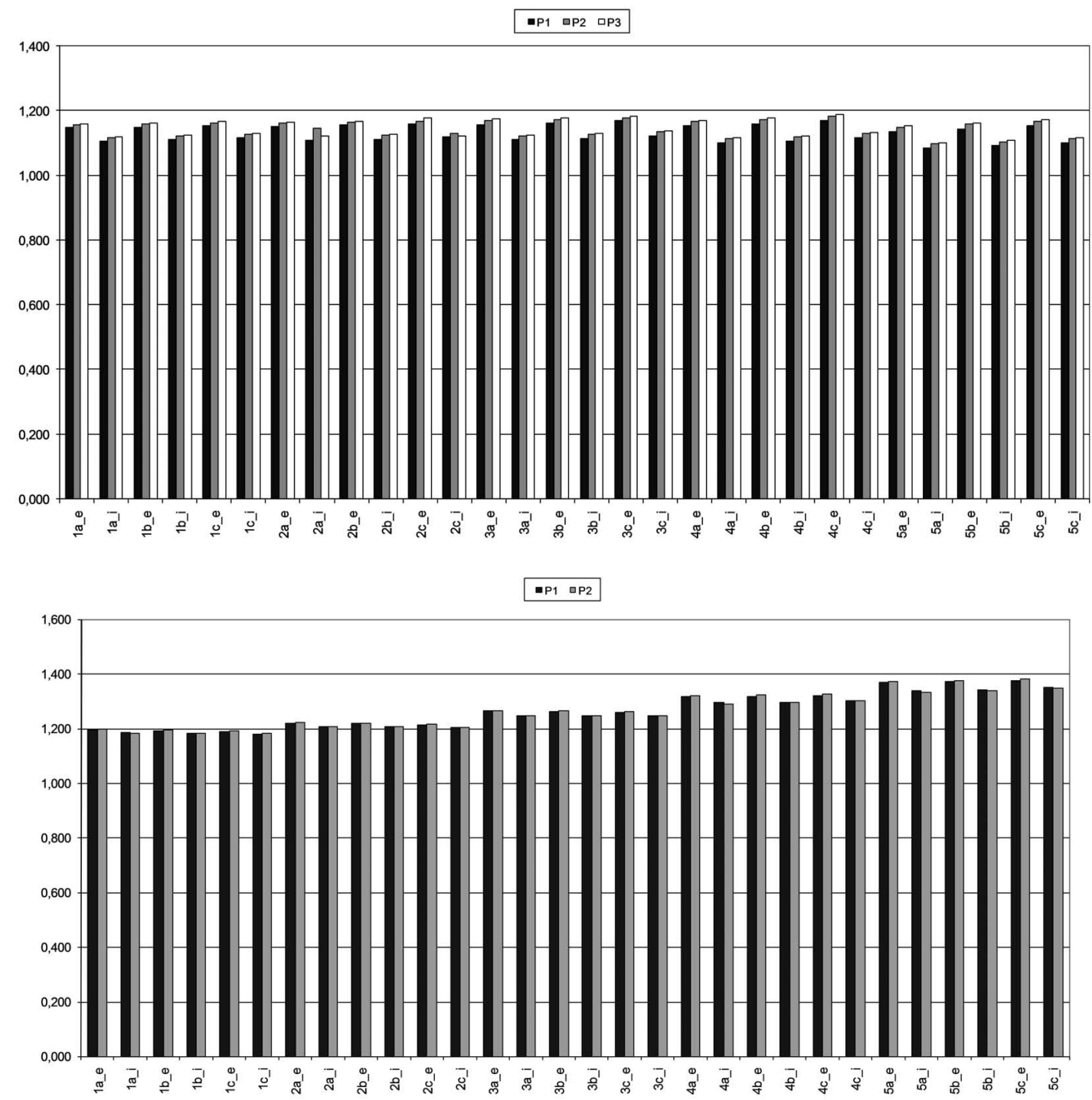


\section{CONCLUSIONES}

La norma española CTE contiene, sustancialmente, los mismos métodos y formulación para cuantificar la carga de viento que EC-1. Sin embargo, se han detectado ciertas divergencias en la aplicación de los métodos desarrollados por la norma europea, principalmente aquellas simplificaciones que introducen ciertas variaciones en la carga de viento:

- Las categorías V de CTE y IV de EC-1 son equivalentes, pues ambas tienen la misma caracterización matemática (iguales valores de la longitud de rugosidad, factor del terreno y altura mínima). Sin embargo, atendiendo a la descripción de cada una, podrían diferir en su interpretación, fundamentalmente a la hora de clasificar un terreno del tipo "Zonas urbanas en general, industriales o forestales".

- La inclusión del tratamiento de efectos dinámicos $\left(C_{s} C_{d}\right)$ en CTE permitiría reducir la carga de viento entre un 3 y un $11,5 \%$, dependiendo del tipo de construcción y de la categoría del terreno.

- Para el modelo de edificio de 25 plantas (en general todo edificio que cumpla $h>$ $2 b)$, prescindir del uso, en el cálculo del coeficiente de exposición, de un perfil de altura de referencia no uniforme con la al- tura, incrementa la carga de viento de CTE más de un $10 \%$ respecto a la de EC- 1 .

- Respecto a las instrucciones IAPF-07 e IAP-11, en la definición de la velocidad de referencia del mapa de viento se detecta una incongruencia en la asignación de la categoría de referencia en el que se encuadran los valores de velocidad. Se describe un terreno de categoría "zona plana y desprotegida frente al viento" que luego se afirma equivalente a categoría II ("zona rural con obstáculos aislados").

- Sería conveniente proporcionar el factor del terreno $k$ redondeado al cuarto decimal, pues al presentarlo redondeado al segundo decimal, en los casos concretos de las categorías IV y $\mathrm{V}$ aparecen incrementos respecto al EC-1 del 4,32\% y del $4,92 \%$, respectivamente.

La mayoría de estas diferencias se han apreciado en los modelos de cálculo del apartado 4. Las consecuencias sobre los modelos, estando la carga de viento combinada apropiadamente con el resto de acciones, son sobrestimaciones de las tensiones equivalentes en la base de los pilares de hasta un $20 \%$ en el modelo de nave industrial, de entre un $10 \%$ y un $17 \%$ en el modelo de edificio de 5 plantas, y de entre un $20 \%$ y un $35 \%$ en el modelo de edificio de 25 plantas.

\section{BIBLIOGRAFÍA}

(1) Comité Técnico AEN/CTN 140: "UNE-EN 1991-1-4 Eurocódigo 1: Acciones en estructuras. Parte 1-4: Acciones generales. Acciones de viento", AENOR, Madrid, 2007.

(2) Goyal, R.: "Wind loads on low-rise buildings - A review of codal specifications", The $21^{\text {st }}$ Century COE Program, Wind Effects on Buildings and Urban Environment, 2007.

(3) Simiu, E.; Sadek, F.; Whalen, T. M.; Jang, L.; Lu, L. W.; Diniz, S. M. C.; Grazini, A.; Riley, M. A.: "Achieving a safer and more economical buildings through database-assisted, reliability-based design for wind", Journal of Wind Engineering and Industrial Aerodynamics, vol. 91, n 12-15 (2003), pp. 1587-1611.

(4) St Pierre, L. M.; Kopp, G. A.; Surry, D.; Ho, T. C. E.: "The UWO contribution to the NIST aerodynamic database for wind loads on low buildings: Part 2. Comparison of data with wind load provisions", Journal of Wind Engineering and Industrial Aerodynamics, vol. 93, n 1 (2005), pp. 31-59.

(5) Kasperski, M.: "Extreme wind load distributions for linear and nonlinear design", Engineering Structures, vol. 14, nº 1 (1992), pp. 27-34.

(6) Ministerio de Vivienda: "Código técnico de la edificación. Documento básico - Seguridad estructural: Acciones en la edificación", Ministerio de Vivienda, Madrid, 2009.

(7) Puertos del Estado: "Recomendaciones para obras marítimas ROM 0.4-95. Acciones climáticas II: Viento", Ministerio de Obras Públicas, Transportes y Medio Ambiente, Madrid, 1995.

(8) Ministerio de Fomento: "Instrucción sobre las acciones a considerar en el proyecto de puentes de carretera (IAP-11)", Ministerio de Fomento, Madrid, 2011.

(9) Ministerio de Fomento: "Instrucción sobre las acciones a considerar en el proyecto de puentes de ferrocarril (IAPF-07)", Ministerio de Fomento, Madrid, 2007.

(10) Ortega, M.; Lacoma, L. M.; Holman, D. M.: "Análisis de las acciones de viento en edificios singulares. Aplicación al hotel Vela de Barcelona", Hormigón y Acero, nº 251 (2009), pp. 37-64.

(11) Morales, M.: "Revisión y estudio comparativo de la acción del viento sobre las estructuras empleando eurocódigo y normativa española", PFC, ETSII, UPCT, Cartagena, 2009.

(12) Garcimartín, M. A.; Sánchez, E.; Larrañeta, M. P.; López, J. F.; Massana, J.: "Propuesta sobre las acciones del viento aplicables en la norma EC-1-13031-1 de invernaderos", Hormigón y Acero, $\mathrm{n}^{\circ} 220$ (2001), pp. 69-76.

(13) Kasperski, M.: "A consistent model for the codification of wind loads", Journal of Wind Engineering and Industrial Aerodynamics, vol. 95, n 9-11 (2007), pp. 1114-1124.

(14) CDyrbye, C.; Hansen, S. O.: Wind loads on structures, John Wiley \& Sons, Chichester, 1999.

(15) Martí, P.; Company, P.; Sanchís, M.: "DISSENY: Un sistema interactivo para el diseño de estructuras basado en técnicas de optimización", Anales de Ingeniería Mecánica, Año 3, no 1 (1985), pp. 285-290.

(16) Martí-Montrull, P.; Company-Calleja, P.: "DISSENY: An integrated system for the structures and structural elements optimal design", Advances in Optimisation for Structural Engineering, B.H.V. Topping (ed.), Civil-Comp Press, Edinburgh, 1996, pp. 23-29. 